\title{
ESTRUTURA FITOSSOCIOLÓGICA DE UM TRECHO DE FLORESTA ESTACIONAL PERENIFÓLIA, BACIA DO RIO DAS PACAS, QUERÊNCIA - MT
}

\author{
Sustanis Horn Kunz'1, Sebastião Venâncio Martins², Natália Macedo Ivanauskas³, Elias Silva², Daniel Stefanello
}

(recebido: 16 de junho de 2009; aceito: 26 de fevereiro de 2010)

\begin{abstract}
RESUMO: A borda sul da região amazônica apresenta um tipo peculiar de floresta, denominada de Floresta Estacional Perenifólia. Diante da falta de conhecimento deste tipo florestal, objetivou-se desenvolver um estudo relacionado à estrutura fitossociológica na bacia do rio das Pacas, em Querência-MT. A amostragem da vegetação consistiu na distribuição de 200 pontos-quadrantes, nos quais foram considerados os quatro indivíduos mais próximos de cada ponto, que tivessem diâmetro à altura de $1,30 \mathrm{~m}$ do solo (DAP) $\geq 10 \mathrm{~cm}$. A densidade total da área amostrada foi 761 ind./ha, distribuídos em 53 espécies, 37 gêneros e 27 famílias. Das espécies de maior Valor de Importância, Ocotea leucoxylon, Myrcia multiflora, Pouteria gardneri, Sloanea eichleri, Miconia pyrifolia e Amaioua guianensis, ocorreram em outros trechos de Floresta Estacional Perenifólia, mas não com a mesma representatividade, evidenciando diferenças estruturais desta unidade fitogeográfica. O índice de Shannon $(3,38)$ pode ser considerado médio e a equabilidade de Pielou $(0,85)$ indica heterogeneidade florística do componente arbóreo.
\end{abstract}

Palavras-chave: Amazônia, diversidade, Xingu.

\section{PHYTOSOCIOLOGICAL STRUCTURE OF A SEAZONAL PERENNIAL FOREST AREA, RIO DAS PACAS BASIN, QUERÊNCIA, STATE OF MATO GROSSO, BRAZIL}

\begin{abstract}
The southern border of the Amazon region presents a peculiar type of forest called Seasonal Evergreen Forest. Due to the lack of knowledge on this type of forest, the objective of this study was to develop a study on the phytosociological structure of rio das Pacas basin vegetation at Querência-MT. The vegetation sampling was composed of the distribution of 200 quadrant-points in which the four individuals closest to each point, with diameter $\geq 10 \mathrm{~cm}$ were considered. The total density of the sampled area was of 761 ind./ha distributed into 53 species, 37 genera and 28 families. From species of high Importance Value, Ocotea leucoxylon, Myrcia multiflora, Pouteria gardneri, Sloanea eichleri, Miconia pyrifolia and Amaioua guianensis had ocurred in other Seazonal Perennial Forest areas, evidencing structural differences of this phytogeographic unit. The Shannon index (3.38) may be considered medium and the Pielou equability (0.85) indicates floristic heterogeneity of the arborous component.
\end{abstract}

Key words: Amazonian, diversity, Xingu.

\section{INTRODUÇÃO}

As inúmeras fitofisionomias da Floresta Amazônica devem-se aos diversos tipos de substrato sob as mesmas, bem como as variações no regime de chuvas. Assim, têmse as florestas de várzea, as florestas de igapó e as florestas de terra-firme (PIRES-O'BRIEN \& O'BRIEN, 1995), sendo que esta última apresenta diversidade florística maior que os demais ecossistemas do mundo (LEITÃO-FILHO, 1987).

A partir de 1980, os estudos florísticos e fitossociológicos na Floresta Amazônica tornaram-se mais frequientes. Contudo, a gama de trabalhos existentes é dedicada, principalmente, ao estado do Amazonas (AMARAL et al., 2000; MATOS \& AMARAL, 1999;
RIBEIRO et al., 1994), Pará (GAMA et al., 2002; LIMAFILHO et al., 2004; SALM, 2004) e Maranhão (MUNIZ et al., 1994a,b). A grande lacuna a respeito da diversidade e mesmo da conservação da flora amazônica é referente à sua porção sul, em sua maior parte no estado de Mato Grosso na área que abrange a bacia do rio Xingu. Nesta região, há extensa área de tensão ecológica entre a Floresta Ombrófila Aberta e a Savana (IBGE, 1993), denominada "ecorregião das florestas secas do Mato Grosso" (FERREIRA et al., 2001), e é onde ocorre a Floresta Estacional Perenifólia (IVANAUSKAS et al., 2008).

Infelizmente, essa formação florestal pouco explorada pelos botânicos está desaparecendo rapidamente, em função das elevadas taxas de

\footnotetext{
${ }^{1}$ Biólogo(a), Mestre em Ciência Florestal - Departamento de Engenharia Florestal/DEF - Universidade Federal de Viçosa/UFV - 36570-000 Viçosa, MG - sustanishk@yahoo.com.br, stefanello.daniel@gmail.com

${ }^{2}$ Engenheiro Florestal, Professor Dr. em Biologia Vegetal - Departamento de Engenharia Florestal/DEF - Universidade Federal de Viçosa/UFV - 36570-000 - Viçosa, MG - venancio@ufv.br, eshamir@ufv.br

${ }^{3}$ Engenheira Agrônoma, Pesquisadora Dra. em Biologia Vegetal - Instituto Florestal do Estado de São Paulo - Divisão de Dasonomia Seção de Ecologia Florestal - Rua do Horto, 931 - Horto Florestal - 02377-000 - São Paulo, SP - nivanaus@yahoo.com.br
} 
desmatamento pela atividade agropecuária. Entre os anos 2004 e 2005, cerca de $12 \%$ do desmatamento total no estado de Mato Grosso aconteceram na Bacia do Xingu (INSTITUTO SOCIOAMBIENTAL, 2009), a qual até 2005 já havia perdido $32 \%$ de sua floresta original (SOUZAJÚNIOR et al., 2006).

Diante disso, neste trabalho objetivou-se descrever a estrutura fitossociológica do componente arbóreo de um trecho de Floresta Estacional Perenifólia, bacia do rio das Pacas, Querência - MT, e discutir esta estrutura em relação a outras formações da região amazônica.

\section{MATERIAL E MÉTODOS}

Este estudo foi realizado na Fazenda São Sebastião (11 $57^{\prime} 34^{\prime \prime} S$ e $\left.052^{\circ} 43^{\prime} 53^{\prime \prime} \mathrm{W}\right)$, pertencente à bacia do rio das Pacas (Figura 1), que é uma sub-bacia do rio Xingu. A precipitação pluviométrica média é de $1800 \mathrm{~mm}$, havendo duas estações bem definidas: a chuvosa e a seca (SEPLAN, 2009). O relevo é basicamente plano e os solos na área amostrada são classificados como Latossolo Amarelo e Vermelho-Amarelo (A. N. Rossete, comunicação pessoal).
A fitofisionomia da área é denominada de Floresta Estacional Perenifólia (IVANAUSKAS et al., 2003), pertencente ao domínio amazônico.

O método de amostragem utilizado foi o de pontoquadrantes (DURIGAN, 2003). Foram estabelecidos 200 pontos regularmente distribuídos para o levantamento fitossociológico, ou seja, em duas linhas distantes $100 \mathrm{~m}$ foram alocados 100 pontos distribuídos a cada $15 \mathrm{~m}$. Foram inclusos todos os indivíduos arbóreos que apresentavam diâmetro a altura de $1,30 \mathrm{~m}$ do solo (DAP) $\geq$ a $10 \mathrm{~cm}$. Os indivíduos mortos em pé foram incluídos na amostragem, mas excluídos da análise fitossociológica, já que é uma ferramenta utilizada para analisar a estrutura da comunidade, de acordo com cada espécie presente na mesma e a categoria (mortos) é representada por indivíduos de várias espécies. A identificação do material botânico seguiu o sistema de classificação Angiosperm Phylogeny Group II - APG II (2003). O material botânico foi incorporado no herbário da Coleção Zoobotânica James Alexander Ratter, do Campus Universitário de Nova Xavantina/ UNEMAT (Herbário NX).

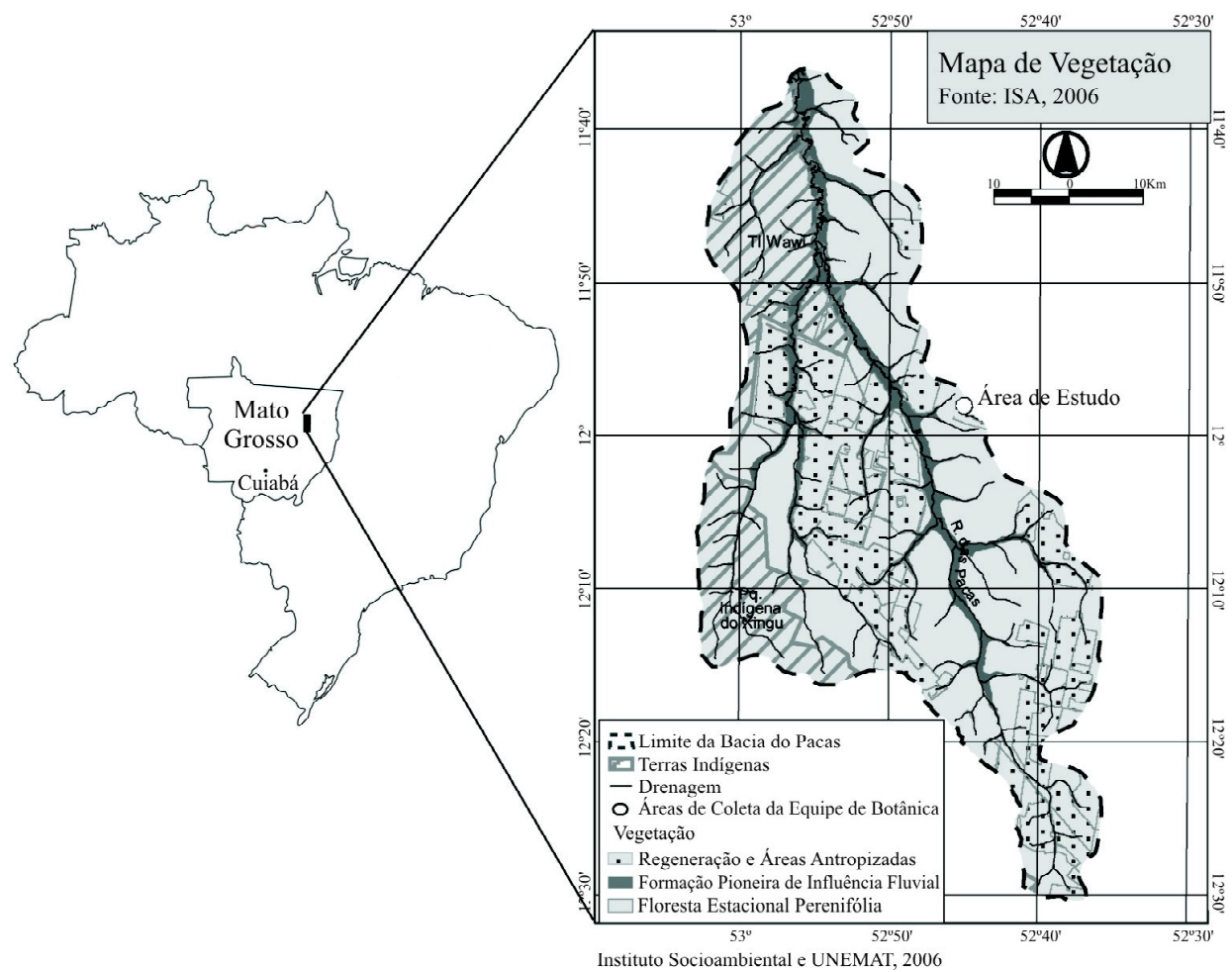

Figura 1 - Localização da bacia do rio das Pacas, com destaque para a Fazenda São Sebastião, município de Querência-MT.

Figure 1 - Localization of Pacas river basin, emphasizing the sampled area in São Sebastião Farm at Querência-MT.

Cerne, Lavras, v. 16, n. 2, p. 115-122, abr./jun. 2010 
Os parâmetros fitossociológicos considerados (Densidade, Frequência, Dominância e Valor de Importância) foram aplicados de acordo com Durigan (2003), pelo programa FITOPAC 1 (SHEPHERD, 1995). Foram analisadas também a diversidade e a equabilidade das espécies na área. Com os dados da listagem de espécies, foi feita uma estimativa de riqueza de espécies por meio do estimador Jackniffe1, permitindo a construção de uma curva de riqueza observada versus estimada.

\section{RESULTADOS E DISCUSSÃO}

A densidade total na área amostrada foi de 771 ind./ ha, considerando os indivíduos mortos. Esse resultado é semelhante ao encontrado em estudos realizados com a mesma metodologia (DAP $\geq 10 \mathrm{~cm}$ a 1,30m do solo) na bacia do Rio Juruá-AM (668 a 862 ind./ha ${ }^{-1}$ ) (SILVA et al., 1992), na bacia do Rio Uatumã-AM (741 ind./ha ${ }^{-1}$, AMARAL et al., 2000) e em um trecho de floresta de terra firme em ManausAM (771 ind./ha ${ }^{-1}$ ) (OLIVEIRA \& AMARAL, 2004). No entanto, apresentou densidade maior quando comparado com trechos de Floresta Estacional Perenifólia em Gaúcha do Norte - MT, onde foram amostrados 546 ind./ha ${ }^{-1}$ (IVANAUSKAS et al., 2004). O valor da área basal $\left(25,80 \mathrm{~m}^{2} /\right.$ ha), considerando os indivíduos mortos, demonstra que a comunidade apresenta grande quantidade de indivíduos finos. Apesar disso, foi maior que aqueles registrados nos trechos de Floresta Estacional Perenifólia de Gaúcha do Norte (18,63 a 23,95 m²/ha) (IVANAUSKAS et al., 2004).
A riqueza estimada para o trecho de floresta na Fazenda São Sebastião foi de 63 espécies (com intervalo de confiança $=6,05)$, superior à riqueza observada $(53$ espécies). Desta forma, apesar de a demarcação de 200 pontos de amostragem corresponder a quase um hectare (0,98 ha), a curva de acumulação de espécies não se estabilizou completamente (Figura 2), sugerindo que o aumento em número de pontos de amostragem poderia indicar uma maior riqueza florística.

Essa situação demonstra que apenas um hectare não é suficiente para amostrar com representatividade a vegetação amazônica, confirmando outros estudos florísticos realizados em trechos de Floresta Amazônica (HAUGAASEN \& PERES, 2006; MUNIZ et al., 1994b; OLIVEIRA \& AMARAL, 2004). Contudo, amostragens inferiores a esta são utilizadas em estudos em Floresta Amazônica (ANDERSON et al., 1995; MACEDO \& ANDERSON, 1993) e, embora nem sempre seja suficiente para florestas do domínio amazônico, contribuem para o conhecimento florístico e estrutural dessas comunidades ainda pouco estudadas, como é o caso da Floresta Estacional Perenifólia, em razão da dificuldade de acesso e localização.

Foram registradas 53 espécies, 37 gêneros e 27 famílias (Tabela 1), sendo que uma espécie foi identificada apenas no nível de gênero. As dez espécies com os maiores Valores de Importância (VI) representaram 55,53\% do VI total.

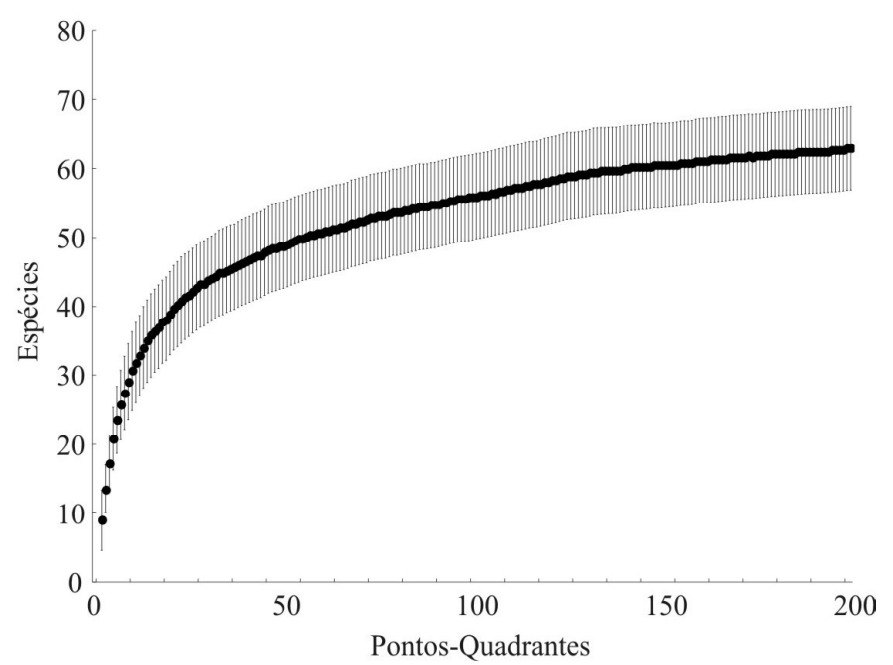

Figura 2 - Curva de riqueza de espécies estimada de um trecho de Floresta Estacional Perenifólia, Querência-MT.

Figure 2 - Species richness curve of sampled area in Seasonal Evergreen Forest in Querencia-MT. 
Tabela 1 - Parâmetros fitossociológicos das espécies arbóreas amostradas no levantamento fitossociológico realizado em QuerênciaMT. NI - Número de indivíduos, DR - Densidade relativa (\%), DoR - Dominância relativa (\%), FR - Frequiência relativa (\%), VI Valor de Importância.

Table 1 - Phytosociological parameters of arborous species sampled in the phytosociological survey performed in Querência-MT. NI - Number of Individuals, DR - Relative Density (\%), DoR - Relative Dominance (\%), FR - Relative Frequency (\%), VI Importance Value.

\begin{tabular}{|c|c|c|c|c|c|c|}
\hline Espécies & Famílias & NI & DR & DoR & FR & VI \\
\hline Ocotea leucoxylon (Sw.) Laness. & Lauraceae & 76 & 10,20 & 19,89 & 9,91 & 40,00 \\
\hline Myrcia multiflora (Lam.) DC. & Myrtaceae & 72 & 9,66 & 6,45 & 7,99 & 24,10 \\
\hline Pouteria gardneri (Mart. \& Miq.) Baehni & Sapotaceae & 45 & 6,04 & 6,90 & 5,92 & 18,86 \\
\hline Miconia pyrifolia Naudin & Melastomataceae & 43 & 5,78 & 3,35 & 5,47 & 14,60 \\
\hline Sloanea eichleri $\mathrm{K}$. Schum. & Elaeocarpaceae & 41 & 5,51 & 3,36 & 5,47 & 14,34 \\
\hline Amaioua guianensis Aubl. & Rubiaceae & 41 & 5,50 & 1,98 & 5,62 & 13,10 \\
\hline Vochysia ferruginea Mart. & Vochysiaceae & 25 & 3,36 & 4,13 & 3,70 & 11,19 \\
\hline Xylopia amazonica R.E. Fr. & Annonaceae & 25 & 3,35 & 3,70 & 3,40 & 10,45 \\
\hline Protium pilosissimum Engl. & Burseraceae & 32 & 4,30 & 1,81 & 4,29 & 10,40 \\
\hline Ouratea discophora Ducke & Ochnaceae & 27 & 3,62 & 2,23 & 3,70 & 9,55 \\
\hline Trattinnickia glaziovii Swart & Burseraceae & 23 & 3,08 & 2,75 & 3,11 & 8,94 \\
\hline Ocotea guianensis Aubl. & Lauraceae & 18 & 2,42 & 3,92 & 2,22 & 8,56 \\
\hline Sacoglottis guianensis Benth. & Humiriaceae & 19 & 2,56 & 3,33 & 2,66 & 8,55 \\
\hline Pseudolmedia macrophylla Trécul & Moraceae & 21 & 2,82 & 1,70 & 3,11 & 7,63 \\
\hline Guatteria schomburgkiana Mart. & Annonaceae & 22 & 2,95 & 1,47 & 3,11 & 7,53 \\
\hline Hirtella gracilipes (Hook. f.) Prance & Chrysobalanaceae & 20 & 2,69 & 2,33 & 2,37 & 7,39 \\
\hline Micropholis egensis (A. DC.) Pierre & Sapotaceae & 18 & 2,41 & 1,94 & 2,66 & 7,01 \\
\hline Matayba guianensis Aubl. & Sapindaceae & 18 & 2,42 & 1,34 & 2,51 & 6,27 \\
\hline Ocotea caudata (Nees) Mez & Lauraceae & 11 & 1,48 & 1,56 & 1,48 & 4,52 \\
\hline Protium unifoliolatum Engl. & Burseraceae & 14 & 1,89 & 0,61 & 1,92 & 4,42 \\
\hline Trattinnickia burserifolia Mart. & Burseraceae & 4 & 0,54 & 3,26 & 0,59 & 4,39 \\
\hline Nectandra sp. & Lauraceae & 11 & 1,47 & 1,14 & 1,63 & 4,24 \\
\hline Miconia dispar Benth. & Melastomataceae & 10 & 1,34 & 1,31 & 1,33 & 3,98 \\
\hline Connarus perrottetii (DC.) Planch. & Connaraceae & 7 & 0,93 & 1,79 & 1,04 & 3,76 \\
\hline Miconia punctata (Desr.) D. Don ex DC. & Melastomataceae & 11 & 1,48 & 0,60 & 1,63 & 3,71 \\
\hline Aspidosperma discolor A. DC. & Apocynaceae & 6 & 0,81 & 1,94 & 0,89 & 3,64 \\
\hline Licania sothersiae Prance & Chrysobalanaceae & 10 & 1,35 & 0,92 & 1,33 & 3,60 \\
\hline Trattinnickia rhoifolia Willd. & Burseraceae & 8 & 1,08 & 1,30 & 1,18 & 3,56 \\
\hline Sacoglottis mattogrossensis Malme & Humiriaceae & 9 & 1,22 & 1,01 & 1,18 & 3,41 \\
\hline Himatanthus sucuиba (Spruce ex Müll. Arg.) Woodson & Apocynaceae & 3 & 0,41 & 2,11 & 0,44 & 2,96 \\
\hline Cheiloclinium cognatum (Miers) A.C. Sm. & Celastraceae & 8 & 1,08 & 0,45 & 1,18 & 2,71 \\
\hline Myrcia amazonica DC. & Myrtaceae & 7 & 0,94 & 0,57 & 1,04 & 2,55 \\
\hline
\end{tabular}

To be continued...

Cerne, Lavras, v. 16, n. 2, p. 115-122, abr./jun. 2010 
Tabela 1 - Continua...

Table 1 - Continued...

\begin{tabular}{|c|c|c|c|c|c|c|}
\hline Espécies & Famílias & $\mathrm{NI}$ & DR & DoR & FR & VI \\
\hline Enterolobium schomburgkii (Benth.) Benth. & Fabaceae & 1 & 0,13 & 1,95 & 0,15 & 2,23 \\
\hline Buchenavia capitata (Vahl) Eichler & Combretaceae & 3 & 0,41 & 1,23 & 0,44 & 2,08 \\
\hline Abuta grandifolia (Mart.) Sandwith & Menispermaceae & 5 & 0,67 & 0,29 & 0,74 & 1,70 \\
\hline Licania gardneri (Hook. f.) Fritsch & Chrysobalanaceae & 2 & 0,26 & 1,01 & 0,30 & 1,57 \\
\hline Xylopia sericea A. St.-Hil. & Annonaceae & 3 & 0,40 & 0,59 & 0,44 & 1,43 \\
\hline Copaifera langsdorffii Desf. & Fabaceae & 2 & 0,26 & 0,79 & 0,30 & 1,35 \\
\hline Mabea fistulifera Mart. & Euphorbiaceae & 3 & 0,41 & 0,29 & 0,44 & 1,14 \\
\hline Hirtella racemosa Lam. & Chrysobalanaceae & 2 & 0,27 & 0,52 & 0,30 & 1,09 \\
\hline Miconia gratissima Benth. ex Triana & Melastomataceae & 3 & 0,41 & 0,12 & 0,44 & 0,97 \\
\hline Trichilia micrantha Benth. & Meliaceae & 2 & 0,26 & 0,38 & 0,30 & 0,94 \\
\hline Inga heterophylla Willd. & Fabaceae & 1 & 0,13 & 0,61 & 0,15 & 0,89 \\
\hline Myrcia fallax (Rich.) DC. & Myrtaceae & 2 & 0,26 & 0,19 & 0,30 & 0,75 \\
\hline Schefflera morototoni (Aubl.) Maguire, Steyerm. \& Frodin & Araliaceae & 2 & 0,27 & 0,14 & 0,30 & 0,71 \\
\hline Pouteria torta (Mart.) Radlk. & Sapotaceae & 2 & 0,26 & 0,11 & 0,30 & 0,67 \\
\hline Maprounea guianensis Aubl. & Euphorbiaceae & 1 & 0,14 & 0,25 & 0,15 & 0,54 \\
\hline Tapirira guianensis Aubl. & Anacardiaceae & 1 & 0,13 & 0,09 & 0,15 & 0,37 \\
\hline Vitex panshiniana Moldenke & Lamiaceae & 1 & 0,13 & 0,08 & 0,15 & 0,36 \\
\hline Duguetia marcgraviana Mart. & Annonaceae & 1 & 0,14 & 0,05 & 0,15 & 0,34 \\
\hline Virola sebifera Aubl. & Myristicaceae & 1 & 0,13 & 0,05 & 0,15 & 0,33 \\
\hline Casearia arborea (Rich.) Urb. & Salicaceae & 1 & 0,13 & 0,05 & 0,15 & 0,33 \\
\hline Matayba arborescens (Aubl.) Radlk. & Sapindaceae & 1 & 0,13 & 0,04 & 0,15 & 0,32 \\
\hline Total & & 745 & 100 & 100 & 100 & 300 \\
\hline
\end{tabular}

As espécies de maior destaque nesse índice foram: Ocotea leucoxylon, Myrcia multiflora, Pouteria gardneri, Miconia pyrifolia e Sloanea eichleri. Oliveira \& Amaral (2004) relatam que as espécies de maior Valor de Importância amostradas em comunidades não perturbadas podem ser utilizadas em planos de manejo em razão da importância ecológica que apresentam, pois os altos valores de dominância, frequência e densidade determinam a representatividade dessas espécies na comunidade, garantindo o equilíbrio do ecossistema.

Ocotea leucoxylon destacou-se neste índice decorrente, principalmente, da alta dominância apresentada. Já Protium pilosissimum, que apresentou valores de densidade e frequência maiores que Vochysia vismiifolia e Xylopia amazonica, ocupou a $9^{\circ}$ posição em função da baixa dominância apresentada. Nos trechos florestais de interflúvio em Gaúcha do Norte-MT, Ocotea leucoxylon, Amaioua guianensis, Miconia pyrifolia, Sloanea eichleri e Xylopia amazonica também estão entre as dez espécies mais importantes na comunidade (IVANAUSKAS et al., 2004). Contudo, as populações apresentam estrutura diferente daquela presente em Querência-MT, a qual varia em relação à dominância, frequência e densidade. Além disso, as demais espécies consideradas importantes no presente estudo em razão da alta frequência, densidade e dominância, foram pouco representativas em Gaúcha do Norte-MT.

Dessa forma, nota-se que apesar de se tratar da mesma formação fitofisionômica, Floresta Estacional Perenifólia, as áreas são estruturalmente diferentes, considerando-se a representatividade das espécies na comunidade. Assim, com o intuito de se conhecer melhor esta formação, seria necessário promover estudos

Cerne, Lavras, v. 16, n. 2, p. 115-122, abr./jun. 2010 
relacionados à similaridade florística, análises da vegetação conjugadas aos fatores ambientais e mais estudos relacionados à fitossociologia, mesmo em locais não muito distantes entre si.

Em nível de gênero, nota-se que apenas Protium (Burseraceae) tem boa representatividade tanto na área do presente estudo como nos trechos de Floresta Ombrófila e Floresta Estacional Perenifólia (IVANAUSKAS et al., 2004; LIMA-FILHO et al., 2004; OLIVEIRA \& AMARAL, 2004, 2005), o que demonstra ampla distribuição desse gênero na região amazônica.

As famílias Burseraceae (5 espécies), Annonaceae (4), Lauraceae (4), Myrtaceae (4) e Melastomataceae (4) representaram $39,6 \%$ da riqueza total (53 espécies). Esta alta representatividade de poucas famílias botânicas para a região amazônica confirma Oliveira \& Amaral (2004), os quais relatam que a alta diversidade florística é representada por poucas famílias. Em outros trechos de Floresta Estacional Perenifólia, as famílias Burseraceae e Lauraceae também se destacam tanto em riqueza como em densidade (IVANAUSKAS et al., 2004), bem como em áreas de Floresta Ombrófila (AMARAL et al., 2000; MATOS \& AMARAL, 1999; OLIVEIRA \& AMARAL, 2004), confirmando a importância das mesmas na flora amazônica.

A diversidade florística está diretamente relacionada com a quantidade de espécies raras presentes na comunidade (ALMEIDA et al., 1993). As espécies consideradas raras na área (um indivíduo por hectare) representaram $17 \%$ do total de espécies, valor bem abaixo daqueles comumente encontrados em trechos de Floresta Amazônica (MUNIZ et al., 1994b; OLIVEIRA \& AMARAL, 2004). Apesar disso, o índice de Shannon (H') encontrado foi 3,38 e a equabilidade de Pielou (J) de 0,85. A Floresta Estacional Perenifólia ocorre em um ambiente físico muito homogêneo, com clima estacional sem mudanças abruptas de temperatura, relevo praticamente plano e solo predominantemente Latossolo Vermelho-Amarelo. Por isso, os valores de diversidade e equabilidade da área analisada podem ser considerados médios, pois nos trechos de Floresta Estacional Perenifólia em Gaúcha do Norte-MT, a diversidade variou de 3,07 a 3,30 e a equabilidade de 0,76 a 0,83 (IVANAUSKAS et al., 2004).

\section{CONCLUSÕES}

A área analisada apresenta-se distinta estruturalmente das demais áreas de Floresta Amazônica. Mesmo considerando a mesma unidade fitogeográfica, as diferenças em relação à estrutura e diversidade florística são muito acentuadas e existem poucos estudos para que uma conclusão mais concreta seja feita a respeito de tais diferenças. No entanto, admite-se a idéia de que a Floresta Estacional Perenifólia apresenta-se como um mosaico vegetacional, onde a comunidade assume diferentes variações estruturais ao longo de um ambiente físico considerado homogêneo.

A fragmentação e o desmatamento acelerado já é uma realidade preocupante naquela região, mesmo antes de se ter conhecimento prévio acerca dos padrões ecológicos e de distribuição das espécies vegetais. Nesse contexto, o desenvolvimento de mais estudos nesta unidade fitogeográfica é necessário, pois facilita o direcionamento na formulação de planos de manejo e contribui para a recuperação de áreas degradadas.

\section{AGRADECIMENTOS}

Agradecemos à USAID, Consórcio Estradas Verdes e FAPEMAT (Processo 08/2004) pelo apoio financeiro para o desenvolvimento do Projeto Gestão Ambiental e Ordenamento Territorial da Bacia do rio Suiá-Miçu. Ao Programa Xingu/ISA e UNEMAT pelo apoio técnicocientífico e logístico. Ao CNPq pela concessão da bolsa de Mestrado à primeira autora. Aos funcionários da Fazenda São Sebastião pelo apoio para a realização deste estudo.

\section{REFERÊNCIAS BIBLIOGRÁFICAS}

ALMEIDA, S. S.; LISBOA, P. L. B.; SILVA, A. S. L. Diversidade florística de uma comunidade arbórea na Estação Científica "Ferreira Penna", em Caxiuanã (Pará). Boletim do Museu Paraense Emílio Goeldi, Série Botânica, Belém, v. 9, n. 1, p. 93-128, 1993.

AMARAL, I. L.; MATOS, F. D.; LIMA, J. Composição florística e parâmetros estruturais de um hectare de floresta densa de terra firme no rio Uatumã, Amazônia, Brasil. Acta Amazonica, Manaus, v. 30, n. 3, p. 377-392, 2000.

ANDERSON, A. B.; MAGEE, P.; GÉLY, A.; JARDIM, M. A. G. Forest management patterns in the floodplain of the Amazon Estuary. Conservation Biology, Essex, v. 9, n. 1, p. 47-61, 1995.

ANGIOSPERM PHYLOGENY GROUP II. An update of the Angiosperm Phylogeny Group classification for the orders and families of flowering plants: APG II. Botanical Journal of the Linnean Society, London, v. 141, p. 399-436, 2003. 
DURIGAN, G. Métodos para análise de vegetação arbórea. In: CULLEN-JÚNIOR, L.; RUDRAN, R.; VALLADARES-PADUA, C. (Orgs.). Métodos de estudos em biologia da conservação e manejo da vida silvestre. Curitiba: UFPR, 2003. p. 455-479.

FERREIRA, L. V.; SÁ, R. L.; BUSCHBACHER, R.; BATMANIAN, G.; SILVA, J. M. C.; ARRUDA, M. B.; MORETTI, E.; SÁ, L. F. S. N.; FALCOMER, J.; BAMPI, M. L. Identificação de áreas prioritárias para a conservação da biodiversidade por meio da representatividade das Unidades de Conservação e tipos de vegetação nas ecorregiões da Amazônia brasileira. In: CAPOBIANCO, J. P. R. (Ed.). Biodiversidade na Amazônia brasileira: avaliação e ações prioritárias para a conservação, uso sustentável e repartição de benefícios. São Paulo: Instituto Socioambiental, 2001. p. 268-286.

GAMA, J. R. V.; BOTELHO, S. A.; BENTES-GAMA, M. de M. Composição florística e estrutura da regeneração natural de floresta secundária de várzea baixa no estuário amazônico. Revista Árvore, Viçosa, v. 26, n. 5, p. 559-566, 2002.

HAUGAASEN, T.; PERES, C. A. Floritisc, edaphic and structural characteristics of flooded and unflooded forests in the lower Rio Purús region of central Amazonia, Brazil. Acta Amazonica, Manaus, v. 36, n. 1, p. 25-36, jan./mar. 2006.

INSTITUTO BRASILEIRO DE GEOGRAFIA E ESTATÍSTICA. Mapa de vegetação do Brasil. Brasília, 1993. Escala 1:5.000.000. Projeção Policônica.

INSTITUTO SOCIOAMBIEnTAL. Desmatamento na Amazônia e agronegócio. Disponível em: <http:// iwww.socioambiental.org/nsa/detalhe?id=23 357 y. Acesso em: 26 mar. 2009.

IVANAUSKAS, N. M.; MONTEIRO, R.; RODRIGUES, R. R. Relações florísticas entre florestas deciduais, semideciduais e perenifólias na região Centro-Oeste do Brasil. In: CLAUDINOSALES, V. (Org.). Ecossistemas brasileiros: manejo e conservação. Fortaleza: Expressão, 2003. p. 313-322.

IVANAUSKAS, N. M.; MONTEIRO, R.; RODRIGUES, R. R. Estrutura de um trecho de floresta amazônica na bacia do alto rio Xingu. Acta Amazonica, Manaus, v. 34, n. 2, p. 275-299, abr./ jun. 2004.

IVANAUSKAS, N. M.; MONTEIRO, R.; RODRIGUES, R. R. Classificação fitogeográfica das florestas do Alto Rio Xingu. Acta Amazonica, Manaus, v. 38, n. 3, p. 387-402, 2008.
LEITÃO-FILHO, H. F. Considerações sobre a florística de florestas tropicais e sub-tropicais do Brasil. IPEF, Piracicaba, $\mathrm{n}$. 35, p. 41-46, abr. 1987.

LIMA-FILHO, D. A.; REVILLA, J.; AMARAL, I. L.; MATOS, F. D. A.; COÊLHO, L. S.; RAMOS, J. F.; SILVA, G. B.; GUEDES, J. O. Aspectos florísticos de 13 hectares da área de Cachoeira Porteira-PA. Acta Amazonica, Manaus, v. 34, n. 3, p. 415-423, jul./set. 2004.

MACEDO, D. S.; ANDERSON, A. B. Early ecological changes associated with logging in an Amazonian floodplain. Biotropica, Washington, v. 25, n. 2, p. 151-163, 1993.

MATOS, F. D. A.; AMARAL, I. L. Análise ecológica de um hectare em floresta ombrófila densa de terra-firme, Estrada da Várzea, Amazonas, Brasil. Acta Amazonica, Manaus, v. 29, n. 3, p. $365-379,1999$.

MUNIZ, F. H.; CESAR, O.; MONTEIRO, R. Aspectos florísticos quantitativos e comparativos da vegetação arbórea da Reserva Florestal do Sacavém, São Luís, Maranhão (Brasil). Acta Amazonica, Manaus, v. 24, n. 3/4, p. 189-218, 1994a.

MUNIZ, F. H.; CESAR, O.; MONTEIRO, R. Fitossociologia da vegetação arbórea da Reserva Florestal do Sacavém, São Luís, Maranhão (Brasil). Acta Amazonica, Manaus, v. 24, n. 3/4, p. 219-236, 1994b.

OLIVEIRA, A. N.; AMARAL, I. L. Florística e fitossociologia de uma floresta de vertente na Amazônia Central, Amazonas, Brasil. Acta Amazonica, Manaus, v. 34, n. 1, p. 21-34, jan./mar. 2004.

OLIVEIRA, A. N.; AMARAL, I. L. Aspectos florísticos, fitossociológicos e ecológicos de um sub-bosque de terra firme na Amazônia Central, Amazonas, Brasil. Acta Amazonica, Manaus, v. 35, n. 1, p. 1-16, 2005.

PIRES-O' BRIEN, M. J.; O' BRIEN, C. M. Ecologia e modelamento de florestas tropicais. Belém: FCAP, 1995. 400 p.

RIBEIRO, J. E. L. S.; NELSON, B. W.; SILVA, M. F.; MARTINS, L. S. S.; HOPKINS, M. Reserva florestal Ducke: diversidade e composição da flora vascular. Acta Amazonica, Manaus, v. 24, n. 1/2, p. 19-30, 1994.

SALM, R. Tree species diversity in a seasonally-dry forest: the case of the Pinkaití site, in the Kayapó Indigenous Area, southeastern limits of the Amazon. Acta Amazonica, Manaus, v. 34, n. 3, p. 435-443, jul./set. 2004.

Cerne, Lavras, v. 16, n. 2, p. 115-122, abr./jun. 2010 
SEPLAN. Zoneamento: dados secundários: dados secundários do projeto ZSEE: divulga. Disponível em: <http:// lwww.seplan.mt.gov.br/ Acesso em: 28 abr. 2009.

SHEPHERD, G. J. FITOPAC 1: manual do usuário. Campinas: Unicamp, 1995. 93 p.

SILVA, A. S. L.; LISBOA, P. L. B.; MACIEL, U. N. Diversidade florística e estrutura em floresta densa da bacia do rio Juruá-AM. Boletim do Museu Paraense Emílio Goeldi, Série Botânica, Belém, v. 8, n. 2, p. 203-258, 1992.

SOUZA-JÚNIOR, C.; VERÍSSIMO, A.; MICOL, L.; GUIMARÃES, S. Transparência florestal: estado de Mato Grosso. Manaus: Instituto do Homem e Meio Ambiente da Amazônia, 2006. (Boletim Técnico, 5). 